\title{
A LOWER BOUND FOR THE VOLUME OF STRICTLY CONVEX BODIES WITH MANY BOUNDARY LATTICE POINTS
}

BY

GEORGE E. ANDREWS( $(1)$

In a recent paper it was shown that if a strictly convex body $C$ in $n$-dimensional space contains $N$ noncoplanar lattice points (i.e., points with integer coordinates) on its boundary, then

$$
S(C)>k(n) N^{(n+1) / n}
$$

where $S(C)$ denotes the surface area of $C$ and $k(n)>0$ is a constant depending only on $n$ [1]. If $V(C)$ denotes the volume of $C$ and

$$
V(C) \geqq c^{\prime}(n)[S(C)]^{n /(n-1)}
$$

where $c^{\prime}(n)>0$ is a constant depending only on $n$ (which is true if $C$ is an $n$ dimensional sphere for example), then the above theorem implies that

$$
V(C)>k^{\prime}(n) N^{(n+1) /(n-1)}
$$

where $k^{\prime}(n)>0$ is a constant depending only on $n$. The object of this paper is to show that the restriction to

$$
V(C) \geqq c^{\prime}(n)[S(C)]^{n /(n-1)}
$$

is unnecessary. The main result will be the following theorem.

THEOREM. If $C$ is an n-dimensional strictly convex body with $N$ noncoplanar lattice points on its boundary, then

$$
V(C)>\kappa(n) N^{(n+1) /(n-1)}
$$

where $\kappa(n)>0$ depends only on $n$.

This paper will be divided into two sections. In the first section we shall obtain certain elementary but necessary results. In the second section we shall prove the above theorem.

Received by the editors January 25, 1962.

(1) The research on this paper was begun at the University of Cambridge under a Fulbright grant and completed at the University of Pennsylvania under a National Science Foundation Graduate Fellowship.

I wish to thank Dr. H. Groemer for suggesting this problem to me when I was a student at Oregon State University. 
I. In this section we shall be primarily concerned with certain elementary results in $n$-dimensional Euclidean geometry. The following notations and conventions will be used throughout this paper.

At all times the bodies we shall deal with will be considered closed and bounded; however, the cones we shall deal with will of course be unbounded.

We shall call a linear $(n-1)$-dimensional boundary element of an $n$-dimensional polytope or cone a "face."

Given the point $\left(a_{1}, \cdots, a_{n}\right)$ and the direction $\left(A_{1}: \cdots: A_{n}\right)$, the "ray" emanating from $\left(a_{1}, \cdots, a_{n}\right)$ in the direction $\left(A_{1}: \cdots: A_{n}\right)$ is given by the set of points $\left(a_{1}+A_{1} t, \cdots, a_{n}+A_{n} t\right)$ and $t \geqq 0$.

If $H$ is a supporting hyperplane of $C$, a convex body, then the "exterior normal vector" of $H$ is the one on the opposite side of $H$ from $C$. We define an "exterior normal ray" as a ray emanating from some given point of $H$ in the direction of the exterior normal vector.

The symbol $\mathrm{O}$ will always denote the origin.

At all times, $\sigma(n)=\pi^{n / 2} / \Gamma(n / 2+1) ; \pi^{n / 2} / \Gamma(n / 2+1)$ is the volume of the $n$ dimensional unit sphere [7, p. 136].

By $(P o)_{n},(P o)_{n}^{a},(P o)_{n}^{b}, \cdots$, we shall denote $n$-dimensional, convex polytopes $[7$, p. 96$]$.

By $\Theta_{i}$ we shall denote an $n$-dimensional solid angle $\left({ }^{2}\right)$. This symbol will be used to denote both the angle and the measure of the angle, but this should cause no confusion.

We let $\Delta_{i}\left(\Theta_{i}\right)$ be an $n$-dimensional pyramid [7, p. 111] with vertex $\boldsymbol{\alpha}_{i}$, vertex angle $\Theta_{i}$, and an $(n-1)$-dimensional convex polytope $(P o)_{n-1}^{\Delta^{i}}$ as base. We shall call the collection of all faces of $\Delta_{i}\left(\Theta_{i}\right)$ other than $(P o)_{n-1}^{\Delta^{i}}$ the "sides" of $\Delta_{i}\left(\Theta_{i}\right)$. To $\Delta_{i}\left(\Theta_{i}\right)$ we associate a similar pyramid $\delta_{i}\left(\Theta_{i}\right)$ which has vertex $\alpha_{i}$, vertex angle $\Theta_{i}$, and has its base parallel to $(P o)_{n-1}^{\Delta^{t}}$ but is such that the total area of its sides is equal to 1 . Also associated with $\Delta_{i}\left(\Theta_{i}\right)$ is a cone $\mathscr{C}_{i}$ which is the collection of all rays emanating from $\alpha_{i}$ and intersecting $(P o)_{n-1}^{\Delta^{t}}$. We designate by $\tilde{\mathscr{C}}_{i}$ the set of all points $\mathbf{y}$ for which the angle $\angle \mathbf{y} \alpha_{\mathbf{i}} \mathbf{x}$ is at least $\pi / 2$ if $x \in \mathscr{C}_{i}$, and $\tilde{\Theta}_{i}$ will denote the $n$-dimensional angle defined by $\tilde{\mathscr{C}}_{i}$.

If $\Theta$ is an $n$-dimensional solid angle defined by $n$ rays (i.e., these rays make up the edges of $\Theta$ ), then

$$
\sin \Theta=\text { df. }\left|\operatorname{det}\left(c_{j}^{i}\right)\right|
$$

where $c_{j}^{i}$ is the $j$ th direction cosine of the $i$ th ray [7, p. 122].

LEMMA $1 . \sin \Theta \leqq(n-1)$ ! $\Theta$.

Proof. If the point $\alpha$ is the vertex of $\Theta$, then $(1 / n !) \sin \Theta$ is the volume of a simplex with edges of unit length emanating from $\alpha$. Hence the whole of this simplex is contained in the body that the cone defined by $\Theta$ cuts out of the unit

(2) The interiors of the solid angles we shall consider will always be contained in half-spaces. 
sphere with center at $\alpha$. The measure of the volume common to this cone and the unit sphere is $\Theta / n$. Therefore

or

$$
\frac{1}{n !} \sin \Theta \leqq \frac{\Theta}{n},
$$

$$
\sin \Theta \leqq(n-1) ! \Theta \text {. }
$$

q.e.d.

The following lemma is most likely due to Mahler [6] and is very similar to Theorem V of Dr. Cassels' book [3, p. 117]. Unfortunately the author was not able to find this result in readily available literature, and it is thus included for completeness.

Leмma 2. Let $C$ be a closed convex body with volume $V(C)>0$. Then there exist points $\mathbf{x}_{0}, \cdots, \mathbf{x}_{n} \in C$ such that $C$ contains the simplex $\mathscr{D}_{n}$ with $\mathbf{x}_{0}, \cdots, \mathbf{x}_{n}$ as vertices and $C$ is contained in the simplex $\mathscr{B}_{n}$ with vertices

$$
\mathbf{x}_{j}+\sum_{i=0 ; i \neq j}^{n}\left(\mathbf{x}_{i}-\mathbf{x}_{j}\right) \quad j=0, \cdots, n .
$$

Proof. Pick the points $\mathbf{x}_{0}, \cdots, \mathbf{x}_{n}$ so that $V\left(\mathscr{D}_{n}\right)$ is a maximum. Such a choice is possible since $C$ is closed and bounded. By convexity $\mathscr{D}_{n}$ is contained in $C$. We note that $\mathscr{B}_{n}$ is a simplex bounded by $n$ hyperplanes each passing through a vertex of $\mathscr{D}_{n}$ parallel to the opposite face of $\mathscr{D}_{n}$. If $C$ were not contained in $\mathscr{B}_{n}$, then for some face $S_{i}(n-1)$ (containing $x_{i}$ ) of $\mathscr{B}_{n}$ there would be a point $\mathbf{y}$ of $C$ which was on the opposite side of $S_{i}(n-1)$ from $\mathscr{D}_{n}$. Then $\mathbf{x}_{0}, \cdots, \mathbf{x}_{i-1}, \mathbf{y}, \mathbf{x}_{i+1}, \cdots, \mathbf{x}_{n}$ would define a simplex contained in $C$ with a larger volume than $V\left(\mathscr{D}_{n}\right)$, a contradiction. Therefore $C \subset \mathscr{B}_{n}$.

q.e.d.

It is easily verified that $\mathscr{B}_{n}$ and $\mathscr{D}_{n}$ are similar simplexes the ratio of similitude being $(n: 1)$; both $\mathscr{B}$ and $\mathscr{D}_{n}$ have the same centroid, and $V\left(\mathscr{D}_{n}\right)=n^{-n} V\left(\mathscr{B}_{n}\right)$.

COROllary 1. $V\left(\mathscr{D}_{n}\right) \leqq V(C) \leqq n^{n} V\left(\mathscr{D}_{n}\right) ; V\left(\mathscr{B}_{n}\right) \geqq V(C) \geqq n^{-n} V\left(\mathscr{B}_{n}\right)$.

Proof. Clear from Lemma 2 and the fact that $V\left(\mathscr{D}_{n}\right)=n^{-n} V\left(\mathscr{B}_{n}\right)$. q.e.d.

Lemma 3. If $\mathscr{C}_{1} \subset \mathscr{C}_{2}$ and both have $\alpha$ as vertex, then $\tilde{\mathscr{C}}_{2} \subset \tilde{\mathscr{C}}_{1}$.

Proof. If $\mathbf{x} \in \tilde{\mathscr{C}}_{2}$ and $\mathbf{y} \in \mathscr{C}_{1}$, then $\mathbf{y} \in \mathscr{C}_{2}$, and hence $\angle \mathbf{x} \alpha \mathbf{y} \geqq \pi / 2$. Hence $\mathbf{x} \in \tilde{\mathscr{C}}_{1}$.

q.e.d.

LEMMA 4. If an $n$-dimensional cone $\mathscr{C}$ is defined by $n$ rays (i.e., $\mathscr{C}$ is the convex cover of $n$ linearly independent rays emanating from a point $\alpha$ ), then $\tilde{\mathscr{C}}$ is the convex cover of the $n$ rays emanating from $\alpha$ each ray being an exterior normal ray to a face of $\mathscr{C}$.

Proof. Let $\mathscr{C}^{*}$ denote the convex cover of the above mentioned $n$ exterior normal rays of $\mathscr{C}$. Without loss of generality we let $\alpha=O$. Let the defining rays of $\mathscr{C}$ have direction numbers $\left(a_{1}^{i}: \cdots: a_{n}^{i}\right) i=1, \cdots, n$. Then the defining rays of $\mathscr{C}^{*}$ have direction numbers $\varepsilon_{i}\left(A_{1}^{i}: \cdots: A_{n}^{i}\right) i=1, \cdots, n$ where $A_{j}^{i}$ is the cofactor of $a^{i}$ in $\operatorname{det}\left(a_{j}^{i}\right)$ and $\varepsilon_{i}= \pm 1$ is chosen so that $\varepsilon_{i}\left(A_{1}^{i}, \cdots, A_{n}^{i}\right)$ is an exterior normal vector to $\mathscr{C}$. We let $\mathbf{A}^{i}=\varepsilon_{i}\left(A_{1}^{i}, \cdots, A_{n}^{i}\right)$ and $\mathbf{a}^{i}=\left(a_{1}^{i}, \cdots, a_{n}^{i}\right)$. 
If $\mathbf{x} \in \mathscr{C}^{*}$, then $\mathbf{x}=\lambda_{1} \mathbf{A}^{1}+\cdots+\lambda_{n} \mathbf{A}^{n}$ where all $\lambda_{i} \geqq 0$. If $\mathbf{y} \in \mathscr{C}$, then $\mathbf{y} \cdot \mathbf{A}^{i} \leqq 0$; hence $\mathbf{y} \cdot \mathbf{x} \leqq 0$. Thus $\mathbf{x} \in \tilde{\mathscr{C}}$.

If $\mathbf{x} \notin \mathscr{C},{ }^{*}$ then $\mathbf{x}=\lambda_{1}^{\prime} \mathbf{A}^{1}+\cdots+\lambda_{n}^{\prime} \mathbf{A}^{n}$ where at least one of the $\lambda_{i}^{\prime}$ is negative, say $\lambda_{k}^{\prime}$. Then $\mathbf{a}^{k} \cdot \mathbf{A}^{i}=0$ if $i \neq k$ and $\mathbf{a}^{k} \cdot \mathbf{A}^{k}=-\left|\operatorname{det}\left(a_{j}^{i}\right)\right|<0$ since $\mathbf{a}^{k}$ and $\mathbf{A}^{k}$ must lie on opposite sides of the face defining $\mathbf{A}^{k}$. Hence $\mathbf{x} \cdot \mathbf{a}^{k}=\lambda_{k}^{\prime} \mathbf{a}^{k} \cdot \mathbf{A}^{k}>0$, and $\mathbf{a}^{k}$ is a point of $\mathscr{C}$ for which $\angle \mathrm{xOa}^{k}<\pi / 2$. Thus $\mathrm{x} \notin \tilde{\mathscr{C}}$.

Therefore $\mathscr{C}^{*}=\tilde{\mathscr{C}}$.

q.e.d.

LEMMA 5. If $\mathscr{C}$ is defined by $n$ rays as in Lemma 4 and $\Theta$ is the vertex angle of $\mathscr{C}$, then

$$
V[\delta(\Theta)] \leqq\left(\frac{n !}{n^{2 n}}\right)^{1 /(n-1)} \sin ^{1 /(n-1)} \tilde{\Theta} .
$$

Proof. In this case we see that $\delta(\Theta)$ is an $n$-dimensional simplex. For convenience we shall consider the origin $O$ as the vertex of $\mathscr{C}$. The edges of $\delta(\Theta)$ emanating from $\mathrm{O}$ are given by the vectors $\left(a_{1}^{i}, \cdots, a_{n}^{i}\right), i=1, \cdots n$. Now

$$
V[\delta(\Theta)]=\frac{1}{n !}\left|\operatorname{det}\left(a_{j}^{i}\right)\right|,
$$

and by Lemma 4 we know that $\tilde{\Theta}$ is defined by the convex cover of the rays emanating from $\mathrm{O}$ normal to the faces of $\mathscr{C}$; thus

$$
\sin \tilde{\Theta}=\left|\operatorname{det}\left(\frac{A_{j}^{i}}{\left(A_{1}^{i 2}+\cdots+A_{n}^{i 2}\right)^{1 / 2}}\right)\right|=\left\{\prod_{i=1}^{n}\left(A_{1}^{i^{2}}+\cdots+A_{n}^{i^{2}}\right)^{-1 / 2}\right\} \cdot\left|\operatorname{det}\left(A_{j}^{i}\right)\right|
$$

where $A_{j}^{i}$ is the cofactor of $a_{j}^{i}$ in $\operatorname{det}\left(a_{j}^{i}\right)$. By the definition of $\delta(\Theta)$, the area of all of its sides (i.e., excluding its base) equals 1 . Now $[1 /(n-1) !]\left(A_{1}^{i^{2}}+\cdots+A_{n}^{i^{2}}\right)^{1 / 2}$ gives the area of the $i$ th side of $\delta(\Theta)$. Hence

$$
\left[\frac{1}{(n-1) !}\right]^{n} \prod_{i=1}^{n}\left(A_{1}^{i^{2}}+\cdots+A_{n}^{i^{2}}\right)^{1 / 2} \leqq n^{-n}
$$

since $\sum_{i=1}^{n}\left|\xi_{i}\right|=1$ implies that $\max \prod_{i=1}^{n}\left|\xi_{i}\right|=n^{-n}$. Thus

Now

$$
\prod_{i=1}^{n}\left(A_{1}^{i^{2}}+\cdots+A_{n}^{i^{2}}\right)^{-1 / 2} \geqq\left[\frac{n}{(n-1) !}\right]^{n} .
$$

Hence

$$
\left|\operatorname{det}\left(a_{j}^{i}\right)\right| \cdot\left|\operatorname{det}\left(A_{j}^{i}\right)\right|=\left|\operatorname{det}\left(a_{j}^{i}\right)\right|^{n} .
$$

$$
\begin{aligned}
\sin \tilde{\Theta} & \geqq\left(\frac{n}{(n-1) !}\right)^{n}\left|\operatorname{det}\left(A_{j}^{i}\right)\right|=\left(\frac{n}{(n-1) !}\right)^{n}\left|\operatorname{det}\left(a_{j}^{l}\right)\right|^{n-1} \\
& =\left(\frac{n}{(n-1) !}\right)^{n}(n !)^{n-1}(V[\delta(\Theta)])^{n-1} \\
& =\frac{n^{2 n}}{n !}(V[\delta(\Theta)])^{n-1} .
\end{aligned}
$$


Therefore

$$
V[\delta(\Theta)] \leqq\left(\frac{n !}{n^{2 n}}\right)^{1 /(-1)} \sin ^{1 /(n-1)} \tilde{\Theta} .
$$

q.e.d.

LEMMA 6. $V[\delta(\Theta)] \leqq \xi(n) \cdot \tilde{\Theta}^{1 /(n-1)}$ where $\xi(n)$ depends only on $n$.

Proof. In the manner of Lemma 2 we circumscribe the base polytope of $\delta(\Theta)$ with an $(n-1)$-dimensional simplex $\mathscr{B}_{n-1}$, and we inscribe an $(n-1)$-dimensional simplex $\mathscr{D}_{n-1}$. Now $\mathscr{B}_{n-1}$ forms an $n$-dimensional pyramid $\Delta\left(\Theta^{\prime}\right)$ with $\alpha$ the vertex of $\Theta$, and $\Theta^{\prime} \supset \Theta$.

Let us for a moment restrict our attention to $H$, the hyperplane containing $\mathscr{D}_{n-1}, \mathscr{B}_{n-1}$, and the base of $\delta(\Theta)$. As we noted after Lemma $2, \mathscr{B}_{n-1}$ and $\mathscr{D}_{n-1}$ have a common centroid. We now inscribe a $\mathscr{B}_{n-1}^{\prime}$ inside $\mathscr{D}_{n-1}$ in exactly the same manner that $\mathscr{D}_{n-1}$ is inscribed in $\mathscr{B}_{n-1}$. Thus in this case $\mathscr{B}_{n-1}, \mathscr{D}_{n-1}$, and $\mathscr{B}_{n-1}^{\prime}$ all have a common centroid say c. If we shrink each linear dimension of $H$ by a factor of $(n-1)^{-2}$ about the point $\mathrm{c}$, then $\mathscr{B}_{n-1}$ will be transformed into $\mathscr{B}_{n-1}^{\prime}$.

If we now shrink each linear dimension of $n$-dimensional space by a factor of $(n-1)^{-2}$ about the point $\mathbf{c}$, then as above $\mathscr{B}_{n-1}$ will be transformed into $\mathscr{B}_{n-1}^{\prime}$, and $\alpha$ becomes $\mathbf{c}+(n-1)^{-2}(\alpha-\mathbf{c})$ which was originally an interior point of $\delta(\Theta)$. Thus the $n$-dimensional simplex $S^{\prime}(n)$ defined by $\mathscr{B}_{n-1}^{\prime}$ and c $+(n-1)^{-2}(\alpha-c)$ is contained in $\delta(\Theta)$, and $\Delta\left(\Theta^{\prime}\right)$ is similar to $S^{\prime}(n)$ the ratio of similitude being $\left((n-1)^{+2}: 1\right)$. Since $S^{\prime}(n) \subset \delta(\Theta)$, the area of the sides of $S^{\prime}(n)$ must be $\leqq 1$. Thus

$$
V\left[\Delta\left(\Theta^{\prime}\right)\right]=(n-1)^{2 n} V\left[S^{\prime}(n)\right] \leqq(n-1)^{2 n} V\left[\delta\left(\Theta^{\prime}\right)\right] .
$$

By Lemma 5,

$$
V\left[\delta\left(\Theta^{\prime}\right)\right] \leqq\left(\frac{n !}{n^{2 n}}\right)^{1 /(n-1)} \sin ^{1 /(n-1)} \tilde{\Theta}^{\prime}
$$

By Lemmas 1 and 3,

$$
\sin \tilde{\Theta}^{\prime} \leqq(n-1) ! \tilde{\Theta}^{\prime} \leqq(n-1) ! \tilde{\Theta} .
$$

Hence

$$
\begin{aligned}
V[\delta(\Theta)] & \leqq V\left[\Delta\left(\Theta^{\prime}\right)\right] \\
& \leqq(n-1)^{2 n}\left(\frac{n !}{n^{2 n}}\right)^{1 /(n-1)}((n-1) !)^{1 /(n-1)} \tilde{\Theta}^{1 /(n-1)} \\
& =\xi(n) \tilde{\Theta}^{1 /(n-1)} .
\end{aligned}
$$

LEMMA 7. Given any bounded convex body $C$ in $n$-dimensional space with volume $V(C)>0$, there exists an affine transformation $T$ of determinant \pm 1 such that $T: C \rightarrow C^{\prime}$ and 


$$
V(C)=V\left(C^{\prime}\right) \geqq \gamma(n)\left[S\left(C^{\prime}\right)\right]^{n /(n-1)}
$$

where $\gamma(n)$ depends only on $n$.

Proof. As in Lemma 2, we inscribe a simplex $\mathscr{D}_{n}$ in $C$ and circumscribe a simplex $\mathscr{B}_{n}$ with the properties listed in that lemma. We choose our transformation $T$ so that it sends $\mathscr{D}_{n}$ into a simplex $\mathscr{D}_{n}^{\prime}$ of the same volume but with equal edges. Thus since

$$
V\left(\mathscr{D}_{n}\right)=V\left(\mathscr{D}_{n}^{\prime}\right)=|\operatorname{det} T| V\left(\mathscr{D}_{n}\right),
$$

we have $|\operatorname{det} T|=1[4$, p. 6]. Since parallelism and volume are preserved under this transformation, we see that $\mathscr{B}_{n}$ is also transformed into a simplex with equal edges say $\mathscr{B}_{n}^{\prime}$. If $a$ is the length of an edge of $\mathscr{D}_{n}^{\prime}$, then

$$
V\left(\mathscr{D}_{n}^{\prime}\right)=\frac{a^{n}}{n !}\left(\frac{n+1}{2^{n}}\right)^{1 / 2}
$$

[7, p. 126], and thus

$$
S\left(\mathscr{D}_{n}^{\prime}\right)=(n+1)\left(\frac{a^{n-1}}{(n-1) !}\left(\frac{n}{2^{n-1}}\right)^{1 / 2}\right) .
$$

Since $\mathscr{B}^{\prime}$ is similar to $\mathscr{D}_{n}^{\prime}$,

$$
V\left(\mathscr{B}_{n}^{\prime}\right)=\frac{(n a)^{n}}{n !}\left(\frac{n+1}{2^{n}}\right)^{1 / 2},
$$

and

$$
S\left(\mathscr{B}_{n}^{\prime}\right)=(n+1)\left(\frac{(n a)^{n-1}}{(n-1) !}\left(\frac{n}{2^{n-1}}\right)^{1 / 2}\right) .
$$

Since $S\left(\mathscr{B}_{n}^{\prime}\right) \geqq S\left(C^{\prime}\right) \geqq S\left(\mathscr{D}_{n}^{\prime}\right)$ by $[2$, p. 47], and since

$$
V\left(\mathscr{B}^{\prime}\right)=\frac{1}{n !}(n+1)^{1 / 2}\left(\frac{(n-1) !}{(n-1) \sqrt{n}}\right)^{n /(n-1)}\left(S\left(\mathscr{B}_{n}^{\prime}\right)\right)^{n /(n-1)}=c_{1}(n)\left(S\left(\mathscr{B}_{n}^{\prime}\right)\right)^{n /(n-1)},
$$

we obtain

$$
\begin{aligned}
V(C) & =V\left(C^{\prime}\right) \geqq V\left(\mathscr{D}_{n}^{\prime}\right)=n^{-n} V\left(\mathscr{B}_{n}^{\prime}\right)=n^{-n} c_{1}(n)\left(S\left(\mathscr{D}_{n}^{\prime}\right)\right)^{n /(n-1)} \\
& \geqq n^{-n} c_{1}(n)\left(S\left(C^{\prime}\right)\right)^{n /(n-1)} \\
& =\gamma(n)\left(S\left(C^{\prime}\right)\right)^{n /(n-1)}
\end{aligned}
$$

One might be tempted to believe that Lemma 7 and the result contained in [1] together imply the theorem to be proved in $\S$ II; however, the transformation $T$ given above does not necessarily have integral coefficients which it would have to have if the desired implication were to hold.

The following result is undoubtedly well known, but the author was unable to find this result completely proved elsewhere. 
LeMma $8\left({ }^{3}\right)$. Given a polytope $(P o)_{n}$ with vertices $\alpha_{1}, \cdots, \alpha_{M}$, then

$$
\sum_{i=1}^{M} \tilde{\Theta}_{i}=n \sigma(n)=\frac{2 \pi^{n / 2}}{\Gamma n / 2}
$$

where $\tilde{\Theta}_{i}$ is the $n$-dimensional exterior angle at $\boldsymbol{\alpha}_{i}$.

Proof. Our problem is to show that the exterior angles (i.e. $\tilde{\Theta}_{1}, \cdots, \tilde{\Theta}_{M}$ ) of $(\mathrm{Po})_{n}$ completely fill the unit sphere. Let us think of the unit sphere as the totality of line segments of length 2 with midpoint in the origin. First, we show that by translating the exterior angles of $(\mathrm{Po})_{n}$ to the origin we obtain all such segments. This follows almost directly from Theorem IV of [3, p. 115] which states (among other things) that if $\mathscr{K}$ is a bounded convex body then there are precisely two supporting hyperplanes to $\mathscr{K}$ parallel to any given hyperplane $H$.

We now pick a direction $\left(A_{1}: \cdots: A_{n}\right)$. There are two supporting hyperplanes $H_{1}$ and $H_{2}$ to $(P o)_{n}$ with exterior unit normals

$\vec{n}_{1}=\left(A_{1}^{2}+\cdots+A_{n}^{2}\right)^{-1 / 2}\left(A_{1}, \cdots, A_{n}\right)$ and $\vec{n}_{2}=\left(A_{1}^{2}+\cdots+A_{n}\right)^{-1 / 2}\left(-A_{1}, \cdots,-A_{n}\right)$ respectively. Now each of these supporting hyperplanes contains at least one vertex of $(P o)_{n}$ say $\alpha_{i} \subset H_{1}$ and $\alpha_{j} \subset H_{2}$ (since a supporting hyperplane to a closed bounded convex set contains at least one extreme point of the set [4, p. 24]). If we consider $\vec{n}_{1}$ to be emanating from $\alpha_{i}$, we have $\vec{n}_{1} \subset \tilde{\Theta}_{i}$ and similarly $\vec{n}_{2} \subset \tilde{\Theta}_{j}$. Thus after translating the exterior angles of $(\mathrm{Po})_{n}$ so that their vertices coincide with $\mathrm{O}$, we obtain at least one pair of vectors $\left(A_{1}^{2}+\cdots+A_{n}^{2}\right)^{-1 / 2}\left(A_{1}, \cdots, A_{n}\right)$ and $\left(A_{1}^{2}+\cdots+A_{n}^{2}\right)^{-1 / 2}\left(-A_{1}, \cdots,-A_{n}\right)$ which gives the required segment for the direction $\left(A_{1}: \cdots: A_{n}\right)$. Hence

$$
\sum_{i=1}^{M} \tilde{\Theta}_{i} \geqq \frac{2 \pi^{n / 2}}{\Gamma(n / 2)}=n \sigma(n) .
$$

On the other hand, if a certain ray $\rho$ emanating from $\alpha_{i}$ is completely in the interior of $\tilde{\Theta}_{i}$, then $\angle \mathbf{x} \boldsymbol{\alpha}_{i} \mathbf{y}>\pi / 2$ if $\mathbf{y} \in \Theta_{i}$ and $x \in \rho$. Hence the hyperplane $H$ through $\alpha_{i}$ which has this ray as an exterior normal ray is such that $H \cap(P o)_{n}$ $=\alpha_{i}$. By $\left[3\right.$, p. 115], no other supporting hyperplane to $(P o)_{n}$ has an exterior normal ray in this direction. Thus after translating the exterior angles of $(\mathrm{Po})_{n}$ so that their vertices coincide with $\mathrm{O}$, none of the interiors of the various angles intersect. Hence

Therefore

$$
\sum_{i=1}^{M} \tilde{\Theta}_{i} \leqq \frac{2 \pi^{n / 2}}{\Gamma(n / 2)}=n \sigma(n)
$$

$$
\sum_{i=1}^{M} \tilde{\Theta}_{i}=\frac{2 \pi^{n / 2}}{\Gamma(n / 2)}=n \sigma(n) .
$$

(3) For $n=2$, this is simply Gauss's theorem on the curvature integral for polygons. 
II. Having established the necessary properties of polytopes in $\S \mathrm{I}$, we now proceed to the general theorem. We shall restate it here for convenience.

THEOREM. If $C$ is an n-dimensional strictly convex body with $N$ noncoplanar lattice points on its boundary, then

$$
V(C)>\kappa(n) N^{(n+1) /(n-1)}
$$

where $\kappa(n)>0$ depends only on $n$.

Proof. Call the set of $N$ boundary lattice points $B_{a}(N)$. The members of $B_{a}(N)$ are the vertices of a convex polytope entirely in the interior of $C[1$, Lemma 1 , p. 273]. Call this polytope $(P o)_{n}^{a}$. Let us now multiply each dimension of space by 3. This transforms $(P o)_{n}^{a}$ into $(P o)_{n}^{b}$ with $V\left[(P o)_{n}^{a}\right]=3^{-n} V\left[(P o)_{n}^{b}\right]$. Call the set of vertices of $(P o)_{n}^{b}, B_{b}(N)$. Clearly if $\mathbf{p}=\left(p_{1}, \cdots, p_{n}\right)$ is in $B_{b}(N)$, then $p_{i} \equiv 0$ $(\bmod 3)$ for $i=1, \cdots, n$. Suppose $\mathbf{p}_{i}$ is a specific vertex of $(P o)_{n}^{b}$. Note that the convex cover of the set of lattice points $\left\{\mathbf{p}_{i}+\left(\mathbf{p}_{j}-\mathbf{p}_{i}\right) / 3, i \neq j, i=1, \cdots, N\right.$, $j=1, \cdots, N\}$ is a convex polytope $(P o)_{n}^{c}$ contained in $(P o)_{n}^{b}$. To each vertex $\mathbf{p}_{i}$ of $(P o)_{n}^{b}$ there is a corresponding face $(P o)_{n-1}^{i}$ of $(P o)_{n}^{c}$ lying on a hyperplane $H_{i}$ such that $\mathbf{p}_{i}$ lies on the opposite side of $H_{i}$ from all other members of $B_{b}(N)$, and if $i \neq j$, then $(P o)_{n-1}^{i} \neq(P o)_{n-1}^{j}\left[1\right.$, Proof of Lemma 3, p. 274]. Thus $H_{i}$ truncates $(P o)_{n}^{b}$ at the vertex $\mathbf{p}_{i}$. A pyramid $\Delta_{i}\left(\Theta_{i}\right)$ is thus formed at each vertex $\mathbf{p}_{i}$ of $(P o)_{n}^{b}$ where $\mathbf{p}_{i}$ is the vertex and $H \cap(P o)_{n}^{b}$ is the base. These pyramids are all disjoint by construction, and the sides of each pyramid are part of the surface (or boundary) of $(P o)_{n}^{b}$. By construction, $\Delta_{i}\left(\Theta_{i}\right)$ contains in its base an $(n-1)$-dimensional convex polytope with lattice point vertices $\left(q_{s 1}, \cdots, q_{s n}\right) s=1, \cdots m \geqq n$. Thus $\Delta_{i}\left(\Theta_{i}\right)$ must at least contain an $n$-dimensional simplex $S(n)$ with lattice point vertices since $\mathbf{p}_{i}=\left(p_{i 1}, \cdots, p_{i n}\right)$ is a lattice point, and thus

$$
V\left[\Delta_{i}\left(\Theta_{i}\right)\right] \geqq V(S(n))=\frac{1}{n !}\left|\operatorname{det}\left(q_{s t}-p_{i t}\right)\right| \geqq \frac{1}{n !} .
$$

We now apply Lemma 7 to $(P o)_{n}^{b}$ and transform $(P o)_{n}^{b}$ into $(P o)_{n}^{d}$ with

$$
V\left[(P o)_{n}^{b}\right]=V\left[(P o)_{n}^{d}\right]=\gamma(n)\left[S\left[(P o)_{n}^{d}\right]\right]^{/(n-)} .
$$

The pyramids dealt with above are also transformed into new pyramids $\Delta_{i}^{\prime}\left(\Theta_{i}^{\prime}\right)$, and still $V\left[\Delta_{i}^{\prime}\left(\Theta_{i}^{\prime}\right)\right]=V\left[\Delta_{i}\left(\Theta_{i}\right)\right] \geqq 1 / n$ !. Let $\mathscr{S}_{i}$ denote the surface area of the sides of $\Delta_{i}^{\prime}\left(\Theta_{i}^{\prime}\right)$. Then clearly

$$
\mathscr{S}_{i}^{n /(n-1)} \cdot V\left[\delta_{i}^{\prime}\left(\Theta_{i}^{\prime}\right)\right]=V\left[\Delta_{i}^{\prime}\left(\Theta_{i}^{\prime}\right)\right]
$$

since $\delta_{i}^{\prime}\left(\Theta_{i}^{\prime}\right)$ is the pyramid similar to $\Delta_{i}^{\prime}\left(\Theta_{i}^{\prime}\right)$ but such that the total area of its sides is equal to 1 . 
Hence

$$
\begin{aligned}
\left(\frac{V\left[(P o)_{n}^{d}\right]}{\gamma(n)}\right)^{(n-1) / n} & \geqq S\left[(P o)_{n}^{d}\right] \\
\geqq & \sum_{i=1}^{N} \mathscr{S}_{i} \\
= & \sum_{i=1}^{N}\left[\left\{\mathscr{S}_{i} \cdot\left(V\left(\delta_{i}^{\prime}\left(\Theta_{i}^{\prime}\right)\right)\right)^{(n-1) / n}\right\} \frac{1}{\left(V\left(\delta_{i}^{\prime}\left(\Theta_{i}^{\prime}\right)\right)\right)^{(n-1) / n}}\right] \\
\geqq & {\left[\sum_{i=1}^{N}\left\{\mathscr{S}_{i} \cdot\left(V\left(\delta_{i}^{\prime}\left(\Theta_{i}^{\prime}\right)\right)\right)^{(n-1) / n}\right\}^{n /(n+1)}\right]^{(n+1) / n} } \\
& \cdot\left[\sum_{i=1}^{N}\left\{\frac{1}{\left(V\left(\delta_{i}^{\prime}\left(\Theta_{i}^{\prime}\right)\right)\right)^{(n-1) / n}}\right\}^{-n}\right]^{-1 / n}
\end{aligned}
$$

(by Hölder's inequality [5, p. 24])

$$
\begin{aligned}
& =\left[\sum_{i=1}^{N}\left\{V\left(\Delta_{i}^{\prime}\left(\Theta_{i}^{\prime}\right)\right)\right\}^{(n-1) /(n+1)}\right]^{(n+1) / n}\left[\sum_{i=1}^{N}\left(V\left(\delta_{i}^{\prime}\left(\Theta_{i}^{\prime}\right)\right)\right)^{n-1}\right]^{-1 / n} \\
& \geqq\left[\sum_{i=1}^{N}(n !)^{-(n-1) /(n+1)}\right]^{(n+1) / n}\left[\sum_{i=1}^{N}(\xi(n))^{n-1} \tilde{\Theta}_{i}^{\prime}\right]^{-1 / n} \quad \text { (by Lemma 6) } \\
& =n !)^{-(n-1) / n} N^{(n+1) / n}(\xi(n))^{-(n-1) / n}\left(\sum_{i=1}^{N} \tilde{\Theta}_{i}^{\prime}\right)^{-1 /} \\
& =(n !)^{-(n-1) / n} N^{(n+1) / n}(\xi(n))^{-(n-1) / n}\left(\frac{2 \pi^{n / 2}}{\Gamma(n / 2)}\right)^{-1 /} \quad \text { (by Lemma 8) } \\
& =\kappa^{\prime}(n) N^{(n+1) / n} .
\end{aligned}
$$

We now combine these results.

$$
\begin{aligned}
V(C) & \geqq V\left[(P o)_{n}^{a}\right] \\
& =3^{-n} V\left[(P o)_{n}^{b}\right] \\
& =3^{-n} V\left[(P o)_{n}^{d}\right] \\
& \geqq 3^{-n} \gamma(n)\left(\kappa^{\prime}(n)\right)^{n /(n-1)} N^{(n+1) /(n-1)},
\end{aligned}
$$

and letting $\kappa(n)=\left(\frac{1}{2}\right) 3^{-n} \gamma(n)\left(\kappa^{\prime}(n)\right)^{n / n-1}$, we obtain

$$
V(C)>\kappa(n) N^{(n+1) /(n-1)} \text {. }
$$

We now have a new proof for the main result in [1, p. 272].

COROLLARY. If $C$ is an n-dimensional strictly convex body with $N$ noncoplaner lattice points on its boundary, then 


$$
S(C)>c(n) N^{(n+1) / n} .
$$

Proof. By the isoperimetric inequality [4, p. 104],

$$
\begin{aligned}
S(C) & \geqq n(\sigma(n))^{1 / n}[V(C)]^{(n-1) / n} \\
& >n(\sigma(n))^{1 / n}(\kappa(n))^{(n-1) / n} N^{(n+1) / n} \\
& =c(n) N^{(n+1) / n} .
\end{aligned}
$$

\section{BIBLIOGRAPHY}

1. G. E. Andrews, An asymptotic expression for the number of solutions of a general class of Diophantine equations, Trans. Amer. Math. Soc. 99 (1961), 272-277.

2. T. Bonnesen and W. Fenchel, Theorie der konvexen Körper, Chelsea, New York, 1948.

3. J. W. S. Cassels, An introduction to the geometry of numbers, Springer-Verlag, Berlin, 1959.

4. H. G. Eggleston, Convexity, Cambridge Tracts in Mathematics and Mathematical Physics, No. 47, Cambridge Univ. Press, Cambridge, 1958.

5. G. H. Hardy, J. E. Littlewood and G. Pólya, Inequalities, Cambridge Univ. Press, Cambridge, 1952.

6. K. Mahler, The geometry of numbers, Duplicated lectures, Boulder, Colorado, 1950.

7. D. M. Y. Sommerville, An introduction to the geometry of $n$ dimensions, Dover, New York, 1958.

University of Pennsylvania,

Philadelphia, Pennsylvania 